\title{
Evaluation of Mast Cells in Dermal versus Subcutaneous Hemangiomas and Hemangiosarcomas in Dogs
}

\author{
Katherine A. Barber' ${ }^{1}$ Kim M. Newkirk ${ }^{2}$, Ann Reed ${ }^{3}$, Robert L. Donnell ${ }^{2}$ \\ ${ }^{1}$ College of Veterinary Medicine, University of Tennessee, Knoxville, USA \\ ${ }^{2}$ Department of Biomedical and Diagnostic Sciences, College of Veterinary Medicine, University of Tennessee, Knoxville, USA \\ ${ }^{3}$ Research Computing Support, Office of Information Technology, University of Tennessee, Knoxville, USA \\ Email: knewkirk@utk.edu
}

Received February 16, 2012; revised March 9, 2012; accepted March 21, 2012

\begin{abstract}
This study investigated the role of mast cells in canine cutaneous vascular tumors, and is the first such study to distinguish between tumors arising in the dermis versus the subcutis. Mast cell numbers in canine cutaneous hemangiomas (HA) and hemangiosarcomas (HSA) were evaluated to identify a relationship between mast cells, tumor type (HA, HSA), histologic location (dermis, subcutis) and tumor recurrence. One hundred and sixty-seven biopsies from 148 dogs were evaluated. Using only one biopsy from each dog, mast cell counts (MCC) for each tumor $(n=148)$ were obtained by averaging the number of mast cells counted in ten 400× fields. A significant difference in mean MCC was found only between tumor types, with HA having more mast cells than HSA (4.2 \pm 4.2 vs. $2.2 \pm 2.6 ; p<0.001)$. No significant difference in mean MCC existed between tumors that recurred and those that did not. There was no difference in recurrence rate between tumor type or histologic location. Our results indicate that benign HA contain more mast cells than malignant endothelial cell tumors, regardless of histologic location; whether this is a cause or effect relationship remains to be determined.
\end{abstract}

Keywords: Canine; Dermis; Hemangioma; Hemangiosarcoma; Mast Cell; Skin; Subcutaneous

\section{Introduction}

Mast cells are derived from CD34+ pluripotent bone marrow hematopoietic stem cells. Undifferentiated, but committed mast cell precursors from the bone marrow enter the blood and travel to tissues where they differentiate into mature mast cells. Although the exact mechanisms of migration and differentiation of mast cells are largely unknown, stem cell factor, which is produced by endothelial cells, fibroblasts or epithelial cells and is known to promote the differentiation of mast cells likely plays a role in this process [1].

Mast cells are important in hypersensitivity and immune reactions, but also play a role in angiogenesis. Mast cell angiogenic factors such as TNF, bFGF, VEGF, and IL-8 act in part by stimulating endothelial cells to secrete proteases to degrade the existing basement membrane, followed by the proliferation and migration of endothelial cells towards the angiogenic stimulus. Other proteases (chymase, tryptase, MMP-9) released by mast cells assist in the degradation of the extracellular matrix to facilitate the migration of the endothelial cells. Degradation of the extracellular matrix results in release of pro-angiogenic factors (VEGF, bFGF, TGF-beta) and anti-angiogenic factors (factor 4, thrombospondin 1, angiostatin, endosta- tin). Inflammatory cells or stromal cells attracted by or activated by the mast cells, respectively, may also release angiogenic factors to further promote or inhibit angiogenesis [2]. The balance of pro- and anti-angiogenic factors determines the extent of angiogenesis.

In tumors, increased angiogenesis and the resultant increase in blood flow allows more effective nutrient delivery and promotes tumor growth. Both canine [3] and human [4] malignant melanomas contain more mast cells than benign melanocytomas, and the increased number of mast cells is associated with a worse prognosis. This suggests that, because of their pro-angiogenic effects, an increase in mast cell number may be related to the malignancy of a tumor. In contrast, mast cell granules also contain anti-angiogenic factors like thromboxane $\mathrm{A}_{2}$, and mast cell proteases can release anti-angiogenic factors from the extracellular matrix, both of which could prevent vascularization and inhibit tumor growth [2]. Human colorectal tumors with high mast cell counts were found to have a significantly better prognosis, with the 5-year patient survival rate at $59.3 \%$ and $33.3 \%$ for tumors with high and low mast cell densities, respectively [5]. These contradictory roles of mast cells in neoplasms demonstrate the complex and multi-factorial regulatory 
mechanisms involved in controlling tumor growth.

The known role of mast cells in angiogenesis and carcinogenesis raises questions about their role in vascular tumors, which are the result of uncontrolled endothelial cell proliferation. Hemangiosarcoma (HSA) is a malignnant neoplasm of the endothelial cells that line blood vessels; hemangiomas (HA) are the benign counterpart. In dogs, visceral HSA arise most commonly in the spleen and in one study represented $2 \%$ of all biopsy submissions [6]; visceral HA are uncommon. Hemangiosarcomas and HA can also arise in the skin and in one study cutaneous HSA represented 1\% of all biopsy submissions [6]; while cutaneous HA comprise $4.5 \%$ of all skin neoplasms in dogs [7]. Hemangiosarcomas arising in the skin have a more favorable prognosis than those arising in visceral sites [8]. Within the skin, HA and HSA can arise either in the dermis or in the subcutis. Dermal HA and HSA typically occur in lightly pigmented and sparsely haired skin, and therefore are frequently associated with prolonged exposure to ultraviolet (UV) light [9]. In contrast, subcutaneous HA and HSA are not known to be UV-associated.

Mast cells are commonly found in canine cutaneous HA and HSA, but a clear relationship between mast cells and these vascular tumors has not yet been established. One group reported that canine cutaneous HSA had more mast cells than cutaneous HA, but this difference was not statistically significant [10]. Another group investigating both cutaneous and visceral vascular tumors found that canine HA contain more mast cells than HSA [11]. In their report there was no difference between the numbers of mast cells in visceral versus cutaneous tumors; however, the authors did not discriminate between tumors arising in the dermis and the subcutaneous tissue. Since the proposed pathogenesis of dermal and subcutaneous vascular tumors is different, the role of mast cells in tumors arising in these histologic locations may also be distinct. This is the first study to investigate the presence and prognostic significance of mast cells in dermal versus subcutaneous HA and HSA.

\section{Materials and Methods}

Biopsy submissions to the University of Tennessee College of Veterinary Medicine (UTCVM) pathology service between 2005-2006 were searched for canine cutaneous HA or HSA. Slides were reviewed by a boardcertified pathologist (KMN) and classified as dermal or subcutaneous and HA or HSA according to published criteria [7]. Briefly, HA were well circumscribed masses composed of discrete vascular structures lined by a single layer of flattened endothelial cells arranged on fine collagenous septa (Figures 1(A), (C)). HSA were poorly circumscribed lesions with anastomosing trabeculae of collagen resulting in poorly or incompletely formed vascular spaces, which were lined by plump endothelial cells. HSA had a higher nuclear-to-cytoplasmic ratio and occasional mitoses (Figures 1(B), (D)). In both HA and HSA, vascular spaces contained erythrocytes or fibrin thrombi. In cases where features of HA and HSA co-existed, a final diagnosis of HSA was made. From hematoxylin and eosin (HE) stained sections, the number of mast cells in the tumor stroma was counted in ten contiguous $400 \times$ fields per tumor and then averaged. Cases were excluded from further analysis if there were fewer than 5 countable $400 \times$ fields of tumor. Fields were composed entirely of tumor; the adjacent dermis or subcutaneous fat was not included in the fields. In all cases, mast cells were well differentiated and easily countable from $\mathrm{HE}$ sections; therefore special stains (toluidene blue) that highlight mast cells were deemed unnecessary (Figures 1(E), (F)). Patient information including age, breed, and gender was determined from the medical record or biopsy submission form. For cases originating from the UTCVM teaching hospital, the medical records were reviewed for evidence of recurrence or development of additional tumors. For outside submissions, a questionnaire was sent to the referring veterinarian to determine if and when the tumor recurred. Referring veterinarians were also asked to confirm the signalment.

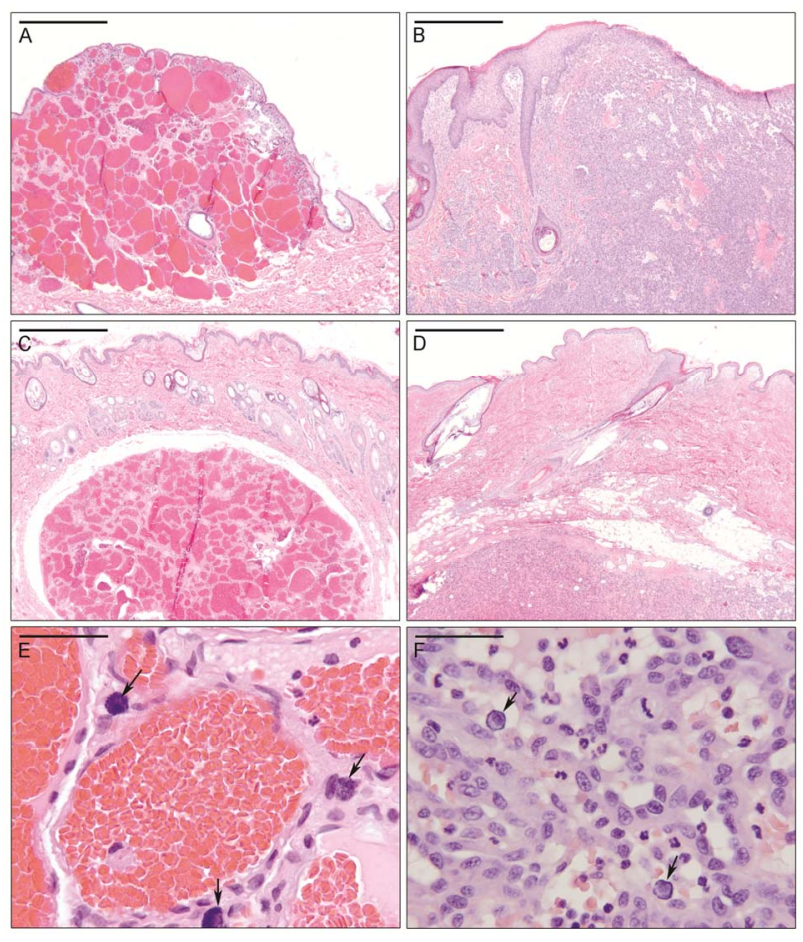

Figure 1. Histologic images of a dermal hemangioma (A); a dermal hemangiosarcoma (B); a subcutaneous hemangioma (C); and a subcutaneous hemangiosarcoma (D); HE. Bars = $2 \mathrm{~mm}$. Mast cells (arrows) in the stroma of a hemangioma (E) and in the stroma of a hemangiosarcoma (F). HE. Bars $=100 \mu \mathrm{m}$. 


\section{Statistics}

Data are reported as mean \pm standard deviation (SD). Associations between the incidence of recurrence, tumor type, and histologic location were evaluated using Fisher's exact test. Mean differences in MCC by gender, tumor type, and histologic location were analyzed with a univariate ANOVA. Normality was assessed using the Shapiro-Wilk W test. Homogeneity of variance was tested using the Levene's test. A log transformation of the data was required for the ANOVA. Mean difference in MCC by recurrence status was tested using an independent samples $t$ test for data with equal variances. Spearman's correlation was used to detect a correlation between MCC and age. For each test, a value of $p<0.05$ was considered significant. Statistical analyses were performed with commercially available software programs.

\section{Results}

Of the 191 biopsies identified as HA or HSA, 167 were included in the study (Table 1). Twenty-four were excluded because they were non-diagnostic, had fewer than 5 countable fields at $400 \times$, or because histologic sections were not available. The 167 biopsies represented 148 dogs; 12 dogs had multiple tumors. One biopsy from each dog was randomly selected to be included in the final data analyses.

The study population included 61 castrated males, 65 spayed females, 14 intact males, and 8 intact females. Breeds most represented included: Golden Retriever ( $\mathrm{n}=$ $17)$, Boxer ( $n=17)$, Labrador Retriever $(n=10)$, Shih-Tzu $(n=5)$, and German Shepherd $(n=5)$; mixed breed dogs, unspecified breeds, and various other breeds with less than five dogs are not listed. The average age of the dogs was 8.9 years, and ranged from 1 to 16 years.

Table 1. Numbers of each tumor type, histologic locations, and recurrences.

\begin{tabular}{cc}
\hline Parameter & Number of Cases (Percent) \\
\hline Tumor type $(\mathbf{n}=\mathbf{1 6 7})$ & $113 / 167(68 \%)$ \\
HA & $54 / 167(32 \%)$ \\
HSA & \\
Histologic location (n = 167) & $102 / 167(61 \%)$ \\
HA & $60 / 102(59 \%)$ \\
HSA & $42 / 102(41 \%)$ \\
Subcutaneous & $65 / 167(39 \%)$ \\
HA & $53 / 65(82 \%)$ \\
HSA & $12 / 65(18 \%)$ \\
Recurrence (n $=\mathbf{6 5})$ & \\
Did not recur & $58 / 65(89 \%)$ \\
Recurred & $7 / 65(11 \%)$ \\
\hline
\end{tabular}

\subsection{Analysis of Mast Cell Count and Individual Tested Parameters}

The mean MCCs and standard deviations for tested parameters (histologic location, tumor type, gender and recurrence) are shown in Figure 2. Mean MCC differed between tumor types, with HA having a significantly higher mean than HSA ( $4.2 \pm 4.2$ vs. $2.2 \pm 2.6$; $p<$ $0.001)$. There was no significant difference in mean MCC between tumors arising in the dermis and those arising in the subcutis, $(p=0.717)$ and there were no significant differences by gender $(p=0.181)$. Additionally there were no significant interactions of tumor type, histologic location or gender ( $p$ ranged from 0.15 to 0.95 ). Mean MCC did not correlate with age $(p=0.237)$ (data not shown).

\subsection{Analysis of Recurrence}

Of the 77 patients with returned questionnaires or follow-up information available in their medical records, only those patients that had been examined at least 1 month after the original diagnosis (65 patients representing 75 tumors) were included in the recurrence analysis. The longest time from diagnosis to follow-up was 4 years. Fifty-eight dogs had no clinical evidence of recurrence at the original biopsy site. Only 7 of the $65(11 \%)$ dogs had a clinically reported recurrence of the tumor at the original biopsy site. The tumor type of the recurred lesions included: 2 dermal HSA, 2 subcutaneous HSA and 3 subcutaneous HA (Table 2). Histologic examination of the surgical margins revealed that of 2 of the 7 tumors that recurred (1 subcutaneous HA and 1 dermal HSA) had been completely excised. Tumors with a diagnosis of HSA were not more likely to recur than HA $(p=0.091)$. There was no significant difference in MCC between those tumors that recurred and those that did not recur ( $p$ $=0.486$ ) (Figure 2). Similarly, the rate of recurrence was not affected by the histologic location (dermal, subcutaneous) ( $p=0.247$ ) (data not shown).

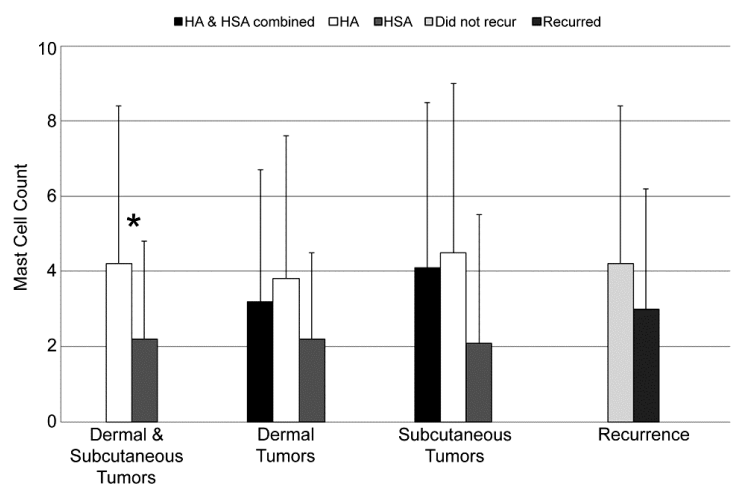

Figure 2. Mean mast cell counts (average number of mast cells in ten $400 \times$ fields) and standard deviations for different tumor types and histologic locations, and as they relate to tumor recurrence. ${ }^{*} p<0.001$. 
Table 2. Incidence of recurrence of tumors based on histologic location and tumor type $(n=75)$.

\begin{tabular}{lccc}
\hline & \multicolumn{2}{c}{ Tumor Type } \\
\hline HA $(\mathrm{n}=\mathbf{5 4})$ & HAS $(\mathbf{n}=\mathbf{2 1})$ \\
\hline $\begin{array}{c}\text { Histologic } \\
\text { Location }\end{array}$ & $\begin{array}{c}\text { Dermal } \\
(\mathbf{n}=\mathbf{4 7})\end{array}$ & 0 & 2 \\
$\begin{array}{c}\text { Subcutaneous } \\
(\mathbf{n}=\mathbf{2 8})\end{array}$ & 3 & 2 \\
\hline
\end{tabular}

\section{Discussion}

Assessing mast cell numbers in various tumor types is an early step in understanding their role in carcinogenesis. To date there are conflicting reports on whether canine cutaneous HA or HSA have more mast cells [10,11]; however, previous reports did not discriminate between tumors arising in the dermis and the subcutaneous tissue. In the current study, HA, regardless of their histologic location in the skin (dermis versus subcutis), consistently had significantly more mast cells than did HSA.

Differing results between these studies are confounded by different methods of staining or counting mast cells. One of the studies created a tissue microarray with a 3 mm core taken from a representative area of the tumor [11], while the other counted mast cells both within the tumor stroma and along the infiltrating edge of the tumor (but found no statistically significant difference in the number of mast cells between the two locations) [10]. In the current report, counting fields were randomly selected and included fields within the center of the tumor and along the periphery of the tumor. Both previous reports $[10,11]$ stained the tissues with toluidine blue to more readily identify the mast cells; as seen in Figures 1(E) and 1(F), the mast cells in the current study were easily counted on routine $\mathrm{HE}$ and toluidine blue staining was not performed. One report used a similar method of counting mast cells [10], but the other report categorized the presence of mast cells (none; few, moderate, or high numbers) [11]. A separate study evaluated the presence or absence of mast cells in canine cutaneous HSA from HE stained sections and found no correlation with the mitotic index, degree of differentiation or clinical outcome [6].

In normal tissues, mast cells are commonly found surrounding blood vessels or nerves, or may be present beneath epithelia [12]. The kinetics behind this localization is unclear, the production of stem cell factor (or other factors) by endothelial cells may partially explain the perivascular location of mast cells in many tissues. In benign HA, these mechanisms may remain intact, whereas malignant endothelial cells in HSA may no longer produce the appropriate mediators required to recruit and promote the differentiation mast cells. Further studies are needed to determine if undifferentiated mast cells, recruited from the circulation by stem cell factor, are present in either of these tumor types; to our knowledge, identification of undifferentiated mast cells is not yet possible in tissue sections.

Additionally, some neoplastic cells express the potent pro-angiogenic factor VEGF, which can also result in mast cell proliferation and recruitment [2]. The findings in the current study do not support VEGF expression as a cause for the increased mast cell numbers, as previous reports have demonstrated increased expression of VEGF in canine HSA while very little VEGF is expressed in HA. It should be noted that that study included visceral and non-visceral HSA, while the HA were exclusively from cutaneous sites [13].

An alternative explanation is that cutaneous sites with higher numbers of mast cells have a protective effect and in the face of endothelial tumorigenesis favor the development of benign lesions. This seems unlikely as the current study found no significant association between mast cell numbers and histologic location. Similarly, when MCC were compared to age, and gender and neuter status, no significant relationships were identified.

Other factors that may affect the number of mast cells in a given tumor is the amount of stroma; since the mast cells are found in the stroma, the MCC would be expected to increase proportionately with the stroma. Although HA tend to have less stroma than HSA, the MCC was still higher in the HA than the HSA. Additionally, as proposed by others, the number of mast cells may change as the tumor evolves [10] or as it is subjected to other stresses (trauma).

Despite the previous reports evaluating mast cells in canine cutaneous vascular tumors, this is the first study to differentiate between tumors arising in the dermis and those arising in the subcutis. In dogs, most vascular tumors arising in the dermis, but not in the subcutis, are known to be UV-associated [9]. Previous studies have suggested that short-haired breeds, particularly the sight hounds, are predisposed to vascular tumors as the short hairs allow for greater UV exposure [6]. In the current study; however, the only short haired breed is the Boxer (11\%). This discrepancy may be due in part to the overall popularity of the retriever dog, which is the most represented of the breeds in this study, with the less common sight hounds (Whippet, Italian Greyhound, Greyhound) representing only $4.7 \%$ of the dogs in this study. In humans and mice, chronic UV exposure is associated with increased numbers of dermal mast cells [14]. Although similar data is not available for dogs, increased numbers of mast cells as a result of chronic UV exposure could promote or inhibit angiogenesis and carcinogenesis. Despite the relationships between UV exposure, mast cells and dermal vascular tumors, there was no significant difference in the MCC of benign or malignant vascular tumors arising in the dermis or subcutis. In future studies, 
evaluation of peri-tumoral mast cells and the presence of other UV-associated changes (solar elastosis) may help provide insight on the pathogenesis of these tumors.

Although more tumors arose in the dermis (61\%), a greater percentage of subcutaneous tumors were benign (HA) (82\% vs. $59 \%$, respectively; $p=0.002)$. Dermal HSA are reported to have a better prognosis than subcutaneous HSA because excision is more likely to be complete $[2,15]$. The reported survival time for subcutaneous HSA varies from 172 to 1189 days depending on the tumor size, presence of metastases at diagnosis, type of chemotherapy, presence of gross disease at the time of treatment [16]. Similar data is not available for dermal HSA. In one report, subcutaneous or intramuscular HSA had a $16 \%$ recurrence rate [16], which is similar to the current study where only $11 \%$ of dogs (for which follow-up was available) had clinical evidence of tumor recurrence. In the current study, neither the histologic location of the tumor (dermis, subcutis) nor the mean MCC was associated with an increased rate of tumor recurrence. Similarly one study evaluating cutaneous HSA found the presence of mast cells had no effect on the clinical outcome [6]. Although HSA, are by definition malignant tumors, in the current study, they were not significantly more likely to recur than HA. Completeness of excision is reported to be the most important factor influencing recurrence of non-visceral HAS [6]. In the current study two of seven tumors that recurred (one subcutaneous HA and one dermal HSA) had been completely excised. Given the small pool of recurrences in the current study, significance differences in factors affecting recurrence may not have been detectable.

It is also important to consider that cutaneous HSA may be arising de novo or could represent metastases from visceral HSA. In one report 7\% of non-visceral HSA were believed to be metastases from visceral HSA [6]. In the current report all tumors were believed to be primary (and not metastatic); this is based in part on the lack of other clinical signs reported in the clinical histories and on follow-up questionnaires.

This study aimed at determining the relevance of mast cells in canine cutaneous HA and HSA, particularly in tumors arising in the dermis versus the subcutis. Regardless of the histologic site of origin, (dermis versus subcutis) HA had significantly more mast cells than HSA. Despite this trend, mast cell counts did not affect recurrence. The role of mast cells in carcinogenesis is complex, and further investigation into the role of mast cells in canine vascular tumors is warranted.

\section{Acknowledgements}

We would like to thank technical communication specialist Misty Bailey, as well as Sharon Schlosshan and Dee Stephenson in the UTCVM histology lab for their assistance with this project.

\section{REFERENCES}

[1] M. A. Scott and S. L. Stockham, "Basophils and Mast Cells,” In: B. V. Fedlman, O. W. Schalm, J. G. Zinkl and N. C. Jain, Eds., Schalm's Veterinary Hematology, 5th Edition, Lippincott Williams \& Wilkins, Baltimore, 2000, pp. 308-317.

[2] K. Norrby, "Mast Cells and Angiogenesis," Acta Pathologica Microbiologica et Immunologica Scandinavica, Vol. 110, No. 5, 2002, pp. 355-371. doi:10.1034/j.1600-0463.2002.100501.x

[3] S. Mukaratirwa, L. Chikafa, R. Dliwayo and N. Moyo, "Mast Cells and Angiogenesis in Canine Melanomas: Malignancy and Clinicopathological Factors," Veterinary Dermatology, Vol. 17, No. 2, 2006, pp. 141-146. doi:10.1111/j.1365-3164.2006.00505.x

[4] L. M. Duncan, L. A. Richards and M. C. Mihn Jr., "Increased Mast Cell Density in Invasive Melanoma," Journal of Cutaneous Pathology, Vol. 25, No. 1, 1998, pp. 11-15. doi:10.1111/j.1600-0560.1998.tb01683.X

[5] S.-Y. Tan, Y. Fan, H.-S. Luo and Z.-X. Shen, "Prognostic Significance of Cell Infiltrations of Immunosurveillance in Colorectal Cancer," World Journal of Gastroenterology, Vol. 11, No. 8, 2005, pp. 1210-1214. doi: $10.1177 / 104063870401600606$

[6] P. Schultheiss, "A Retrospective Study of Visceral and Nonvisceral Hemangiosarcoma and Hemangiomas in Domestic Animals," Journal of Veterinary Diagnostic Investigation, Vol. 16, No. 6, 2004, pp. 522-526.

[7] T. L. Gross, P. J. Ihrke, E. J. Walder and V. K. Affolter, "Skin Diseases of the Dog and Cat: Clinical and Histopathologic Diagnosis," 2nd Edition, Blackwell Science, Oxford, 2006.

[8] M. H. Goldschmidt and M. J. Hendrick, "Tumors of the Skin and Soft Tissues,” In: D. J. Meuren, Ed., Tumors in Domestic Animals, 4th Edition, Iowa State University Press, Ames, 2002, pp. 45-117.

[9] A. M. Hargis, P. J. Ihrke, W. L. Spangler and A. A. Stannard, “A Retrospective Clinicopathologic Study of 212 Dogs with Cutaneous Hemangiomas and Hemangiosarcomas," Veterinary Pathology, Vol. 29, No. 4, 1992, pp. 316-328. doi:10.1177/030098589202900406

[10] M. Woldemeskel and S. Rajeev, "Mast Cells in Canine Cutaneous Hemangioma, Hemangiosarcoma and Mammary Tumors," Veterinary Research Communications, Vol. 34, No. 2, 2010, pp. 153-160. doi:10.1007/s11259-010-9341-1

[11] S. Sabattini and G. Bettini, “An Immunohistochemical Analysis of Canine Haemangioma and Haemangiosarcoma," Journal of Comparative Pathology, Vol. 140, No. 2-3, 2009, pp. 158-168. doi:10.1016/j.jcpa.2008.10.006

[12] A. K. Abbas and A. H. Lichtman, "Cellular and Molecular Immunology,” 5th Edition, Elsevier Science, Philadelphia, 2003.

[13] K. Yonemaru, H. Sakai, M. Murakami, T. Yanai and T. Masegi, "Expression of Vascular Endothelial Growth 
Factor, Basic Fibroblast Growth Factor, and Their Receptors (Flt-1, Flk-1, and Flg-1) in Canine Vascular Tumors," Veterinary Pathology, Vol. 43, No. 6, 2006, pp. 971-980. doi:10.1354/vp.43-6-971

[14] M. A. Grimbaldeston, J. J. Finlay-Jones and P. H. Hart, "Mast Cells in Photodamaged Skin: What Is Their Role in Skin Cancer?” Photochemical and Photobiological Sciences, Vol. 5, No. 2, 2006, pp. 177-183. doi:10.1039/b504344a

[15] H. Ward, L. E. Fox, M. B. Calderwood-Mays, A. S.
Hammer and C. G. Couto, "Cutaneous Hemangiosarcoma in 25 Dogs: A Retrospective Study,” Journal of Veterinary Internal Medicine, Vol. 8, No. 5, 1994, pp. 345-348. doi:10.1111/j.1939-1676.1994.tb03248.x

[16] K.-B. Shiu, A. B. Flory, C. L. Anderson, J. Wypij, C. Saba, H. Wilson, I. Kurzman and R. Chun, "Predictors of Outcome in Dogs with Subcutaneous or Intramuscular Hemangiosarcoma," Journal of the American Veterinary Medical Association, Vol. 238, No. 4, 2011, pp. 472-479. doi:10.2460/javma.238.4.472 